\title{
12 Responding to reviewers
}

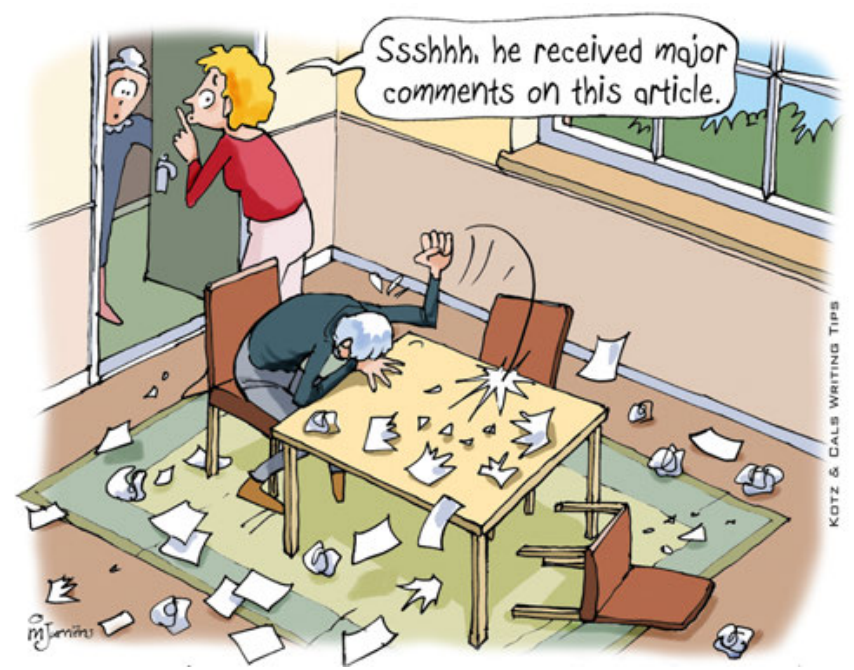

Tip 12 - Responding to reviewers: don't get frustrated!

\section{What you should know}

There are three types of editorial decisions about submitted papers: acceptance, rejection (immediately by the journal's editor or after peer review) or revision (usually with peer review). Many published papers have been rejected and/or revised several times before being accepted. Receiving a "revise and resubmit" decision proves that a journal is interested, which is good news because it means there is a good chance of acceptation if you respond satisfactorily to the reviewers' comments.

Journals experience difficulties in obtaining a sufficient number (at least 2) of high-quality reviewer reports in time. Such reports contain comments from the reviewer to the author (usually anonymously) and additional comments to the editor which will not be forwarded to the author. Reviewers' comments and recommendations frequently differ from each other. Editors will use these reports to judge whether the findings reported in a paper are sufficiently substantiated, but they will also base their decision on their judgement about whether these findings are new and relevant to their audience. 
A "reject after review" decision contains the reviewers' comments on the paper. You can appeal against such a decision, but this appeal should have solid grounds (e.g., a clearly flawed or biased review). A "revise and resubmit" decision contains the reviewers' comments and sometimes additional editorial comments. A well written peer review is structured into "major comments", which you will definitely need to address in a revision, and "minor comments". Each comment ideally includes a clear point of criticism with reference to a specific part of the paper and a suggestion for revision (if possible). The revised version of the paper will be read and judged by the editor and may also be returned to the same reviewers to assess whether comments have been addressed satisfactorily. Reviewers and editors may then ask for further revisions.

\section{What you should do}

Don't panic when receiving a "reject after review" decision! Be aware that papers are more often rejected than accepted. Reviewer reports will give you free advice on how to improve your paper. Once you have received the decision, read it, sleep on it and read it again, reflecting on the reasons for rejection. Share the rejection decision with your co-authors and use the opportunity to further strengthen your manuscript before submitting it to a different journal. Don't leave it too long, and motivate yourself to start this next submission as soon as possible. Be as careful with any new submission of your paper as with the first.

When receiving a "revise and resubmit" decision, read the report carefully and let it sink in before writing the response. Copy/paste all comments into a new document and respond to each comment point-by-point according to the following structure. (1) Author's response: briefly respond to the criticism. (2) Changes to the paper: state whether and where in the paper you have made revisions. Indicate revisions to your paper in the present tense or past perfect, e. g., "We now present data on [...] in Table 1" or "We have added information on [...] to the third paragraph of the methods section". In your response to the reviewers' comments, clearly distinguish your response from the reviewers' comments by choosing a different font, indenting the text, or starting with a marker such as ">”. In your revised paper, mark the text that has been changed since the previous version, e.g., by using the "track changes" option of your word processor. Circulate your responses and the revised paper among the co-

\footnotetext{
* Wir verwenden in diesem Buch das generische Maskulinum und meinen damit auch Wissenschaftlerinnen, Leserinnen etc.
} 
authors, incorporate their feedback and get their approval on the new version before resubmitting to the journal.

Always be respectful towards the reviewers in your response to their comments, even if you find them ridiculous or uninformed. Add a word of thanks to each reviewer for taking the time to suggest improvements and try to adhere to as many suggestions for revision as you can agree with. You can, however, also respectfully disagree with a reviewer's comment. Provide solid arguments to support your point of view, including references to evidence from your own data or from previously published work. Some comments can be addressed in the author's response without making changes to the paper, in particular when there were no specific suggestions for revision by the reviewer. In any case, reviewers reading your response and the revised paper should get the impression that you have taken their comments seriously and that you have done your best to improve the paper accordingly. In the end, you will find that hoped-for email in your inbox saying "accepted for publication". Cherish that moment and be sure to celebrate it!

\section{Checklist for responding to reviewers}

- Provide a point-by-point response to all reviewer comments, structured as:

- author's response to the reviewer (in a respectful tone)

- changes to the paper (whether changes have been made and where)

- Provide a marked revision of your paper (corresponding to the response to reviewers)

- In case of rejection:

- don't get frustrated and motivate yourself to move on,

- improve your paper if possible, based on the reviewers' comments, and

- $\quad$ submit the new version to a different journal quickly

- Get your co-authors' approval on revisions and re-submissions 